\title{
Periodic Fading of VLF Signals Received Over Long Paths During Sunrise and Sunset
}

\author{
D. D. Crombie
}

Contribution From the Central Radio Fropagation Laboratory, National Bureau of Standards, Boulder Laboratories, Boulder, Colo.

\begin{abstract}
Data on the periodic changes in amplitude and phase of VLF signals received over long VLF paths is examined. It is concluded that the variations are due to multimode propagation in the nighttime portion of the path.
\end{abstract}

\section{Introduction}

It has long been known that during sunrise VLF signals received over a long path show periodic variations in amplitude. At sunset similar, though smaller, variations occur. Observations in recent years [Pierce, 1957] of the relative phase delay of such signals show that the amplitude variations are accompanied by phase variations.

The original explanation [Yokoyama and Tanimura, 1933] was that during the day the ionosphere behaves like a metallic reflector having a pseudoBrewster angle near $90^{\circ}$ (grazing incidence), while at night it behaves like a dielectric with the pseudoBrewster angle near $30^{\circ}$. During sunset or sunrise the Brewster angle changes from $30^{\circ}$ to $90^{\circ}$ and at some instant passes through a value equal to the angle of incidence of the wave. The signal level then decreases at this time because of the reduced reflection coefficient. This drop in amplitude of the received signal would then be repeated each time the sunrise (SR) or sunset (SS) line passed each "point" of reflection on a multihop path. This explanation now seems unlikely to be valid since calculations [Wait and Perry, 1957] of the reflection coefficient of the ionosphere for VLF waves show that the decrease in reflection coefficient at the pseudoBrewster minimum is small.

More recently, Rieker [1963] has attempted to explain the periodic phase variations observed during sunrise and sunset at Neuchatel (Switzerland) on the $18 \mathrm{kc} / \mathrm{s}$ signals from NBA in the Canal Zone. His explanation, which is again based on a single ray geometrical optics approach, is that the five "steps" in the sunrise phase variation are due to the SR line advancing over each of the five reflection points of the five hop path. Rieker's explanation does not attempt to account for the amplitude minima which accompany the phase "steps."

Explanations of the sunset and sunrise phenomena which are based on a ray-optics model such as those mentioned above and which invoke only one ray, seem unlikely to be able to account correctly for the observations on long paths for two reasons. The first is, that as has been shown [Budden, 1961; Wait, 1962a], it is necessary to include many rays in explaining VLF propagation over great distances, whereas only a few low order modes are necessary. Secondly, as has been discussed by Wait [1962a], it is necessary to include corrections for diffraction when using ray-optic models on a curved earth.

It is the purpose of this paper to give an account of the fading phenomena observed on two long VLF paths. Following this, an explanation which appears to account for most of the observations will be outlined. This explanation involves interference between the two lowest order modes propagating in the nighttime portion of the earth-ionosphere waveguide.

\section{Experimental Observations}

Observations of the diurnal phase and amplitude variations of the signals from several distant VLF transmitters have been made at the Boulder Laboratories of the National Bureau of Standards and at the Battelle Institute, Frankfurt, Germany, for more than a year. Two paths that are sufficiently long and correctly oriented to show well-developed sunrise and sunset fading with a fairly good signal to noise ratio are

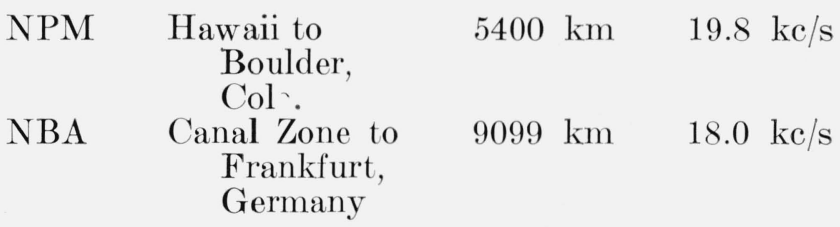

These two paths show effects which are representative of the effects observed on other paths which are nearly perpendicular to the SS or SR line. Thus observations made during 1962 on these two paths will be discussed in detail.

\subsection{Typical Diurnal Variations}

Typical diurnal amplitude and phase records for each of the two paths are shown in figure 1 . These 

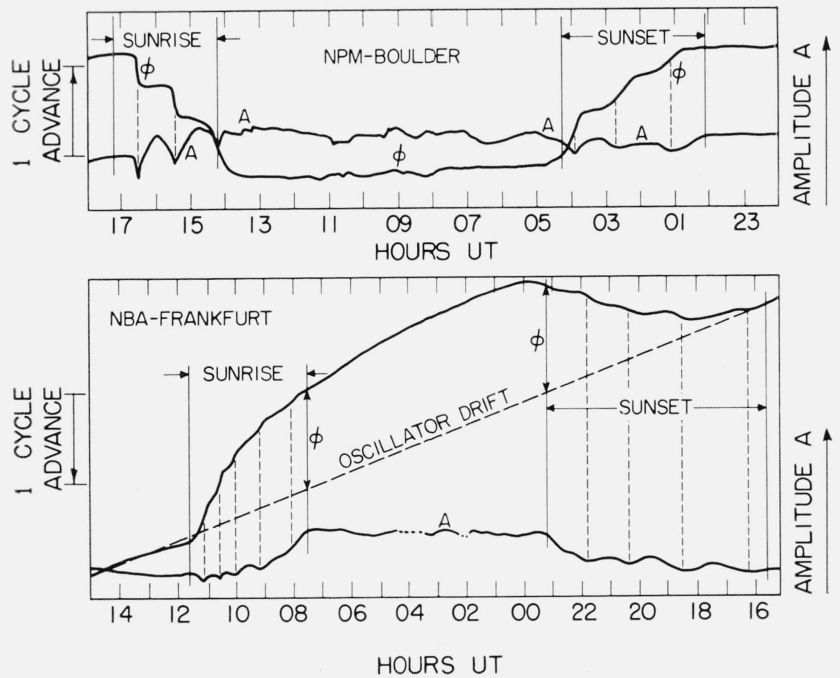

Figure 1. Typical diurnal phase $(\phi)$ and amplitude (A) variations of the signals received from NPM at Boulder and NBA at Frankfurt during January 1962.

have been drawn from the original recordings and some smoothing has been introduced.

These curves are representative of those obtained at other times of the year, except for the times of occurrence of fading and its magnitude. They illustrate most of the features of interest. The first point is that the NPM signal at Boulder shows three amplitude minima during sunrise. The NBA signal at Frankfurt, however, shows evidence of five such minima. Similar but less evident changes occur during sunset. Secondly, it is clear that during sunrise on both paths the amplitude minimum which occurs at the latest time is the deepest. This minimum occurs when the sunrise line is closest to the transmitter (the western end of the path). This is also usually true at sunset on the NPM-Boulder path but is not always the case on the NBA-Frankfurt path. During the northern hemisphere summer the depth of fading at sunset is less than during the winter on both paths, and even in the case of NPMBoulder it cannot be observed during May.

Figure 1 also shows that at the times of amplitude minima the rate of change of phase becomes quite large, for both paths. This relation between amplitude and rate of change of phase appears to hold during all seasons. The same effect is shown in Rieker's [1963] paper.

VLF transmitters frequently change their keying rate and consequently the mean amplitude of received signal varies when observed by a receiver which integrates for a period of several seconds. This frequently makes it difficult to observe small variations in signal level due to propagation changes. Thus it has proved convenient to use the times of occurrence of maximum rate of change of phase to identify the times of minimum signal amplitude, since the rate of change of phase is not affected by changes in the keying rate.

A further important typical feature illustrated in figure 1 is that the rapid changes in phase at the

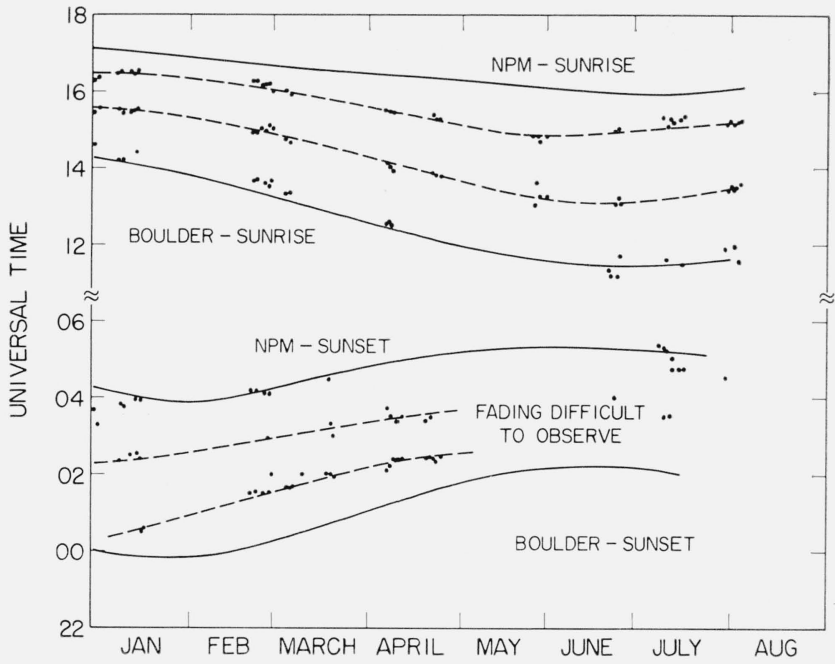

Figure 2. Seasonal variation in time of signal minima for the NPM signal received at Boulder.

times of minimum signal are in the direction of decreasing phase delay during sunrise and of increasing phase delay at sunset. These directions are the same as the overall phase change during these times.

\subsection{Seasonal Variations in Time of Fading}

A further interesting feature of the observations is the regularity (in time) with which the fading occurs. This is demonstrated in figure 2 which is a plot of the times of signal minima on the NPM to Boulder path for the first seven months of 1962 in relation to the times of ground sunrise and sunset. It is clear that the times of sunrise fading repeat themselves with surprising regularity, but that there is a slow seasonal variation in the time at which the fades occur. Figure 2 also shows that fading occurs while the SR or SS line lies between the transmitter and receiver. The regularity of the sunset fading is less than at sunrise, and, as mentioned above, the fading is difficult to observe during the summer months.

In the case of the NBA-Frankfurt path, the times of sunrise fading are also very regular, but sometimes one or two cycles of the fading sequence are too small to be seen. The others, however, seem to occur at the times expected. This is also the case at sunset when it is even more prevalent, presumably because of the small overall depth of fading. No attempt has yet been made to compare the disappearance of fading with geomagnetic or other activity.

Although the times of fading are repeatable from day to day, these times are not particularly meaningful in themselves because of the large seasonal variation in the duration of sunset or sunrise on the path. The times at which signal minima occur can, however, be used to determine the position of the sunrise or sunset line on the path. Figure 3 shows a plot of the monthly average of the change in phase occurring 
in intervals of $5 \mathrm{~min}$ for the NPM-Boulder path during the month of March 1962. Some representative values of the standard deviation of the mean 5min phase differences are also shown as vertical bars. Superimposed on the rate of change of phase curves are curves showing the percentage of the path illuminated at any time. The two curves are for values of $\chi$, the sun's zenith angle, of $90^{\circ}$ and $98^{\circ}$. Thus, at any time the length of illuminated path can be found for either of the $\chi$ values. It is interesting to note from figure 3 that, in this particular case at least, the times between successive signal minima (points of maximum rate of change of phase) are essentially the same whether $\chi$ is taken as $90^{\circ}$ or $98^{\circ}$. This is also found to be approximately the case for both paths at other times of the year. Using diagrams similar to figure 3 the monthly mean distances along the path between signal minima (as deduced from the times) for both sunrise and sunset on the NPM-Boulder path have been obtained and are given in table 1.

TABLE 1. Length of path between successive signal minima, NPM-Boulder 1962

$($ Path length $=5380 \mathrm{~km})$

\begin{tabular}{|c|c|c|c|c|c|c|}
\hline \multirow{3}{*}{1962 month } & \multicolumn{3}{|c|}{ Sunrise } & \multicolumn{3}{|c|}{ Sunset } \\
\hline & \multicolumn{2}{|c|}{$\%$ of path between- } & \multirow{2}{*}{$\begin{array}{l}\text { Mean } \% \\
\text { and dis- } \\
\text { tance } \mathrm{km}\end{array}$} & \multicolumn{2}{|c|}{$\%$ of path between- } & \multirow{2}{*}{$\begin{array}{l}\text { Mean \% } \\
\text { and dis- } \\
\text { tance km }\end{array}$} \\
\hline & $\begin{array}{c}\text { 1st and } \\
2 d \\
\text { minima }\end{array}$ & $\begin{array}{l}2 \mathrm{~d} \text { and } 3 \mathrm{~d} \\
\text { minima }\end{array}$ & & $\begin{array}{l}1 \text { st and } \\
2 \mathrm{~d} \\
\text { minima }\end{array}$ & $\begin{array}{l}2 \mathrm{~d} \text { and } 3 \mathrm{~d} \\
\text { minima }\end{array}$ & \\
\hline Jan_- & 40 & 42 & $41 \%$ & 36 & 36 & $36 \%$ \\
\hline Feb - & 37 & 33 & $\begin{array}{c}2200 \mathrm{~km} \\
35 \%\end{array}$ & 36 & 33 & $\begin{array}{r}1940 \mathrm{~km} \\
34.5 \%\end{array}$ \\
\hline Mar. & 37 & 38 & $\begin{array}{l}1890 \mathrm{~km} \\
37.5 \%\end{array}$ & 40 & 42 & $\begin{array}{c}1850 \mathrm{~km} \\
41 \%\end{array}$ \\
\hline Apr . & 35 & 41 & $\begin{array}{l}2020 \mathrm{Km} \\
38 \%\end{array}$ & 33 & 45 & $\begin{array}{c}2200 \mathrm{~km} \\
39 \% \\
2100 \mathrm{~km}\end{array}$ \\
\hline May.. & 38 & 37 & $37.5 \%$ & $?$ & $?$ & ? \\
\hline June.. & 35 & 41 & $38 \%$ & ? & $?$ & ${ }^{*} 1590 \mathrm{~km}$ \\
\hline July _. & 39 & 42 & $\begin{array}{c}1990 \mathrm{~km} \\
40.5 \% \\
2180 \mathrm{~km}\end{array}$ & ? & $?$ & ${ }^{*} 1630 \mathrm{~km}$ \\
\hline $\mathrm{Aug}$ & 37 & 41 & $\begin{array}{c}39 \% \\
2100 \mathrm{~km}\end{array}$ & 32 & 33 & $32.5 \%$ \\
\hline Sept. & 38 & 43 & $\begin{array}{c}40.5 \% \\
2180 \mathrm{~km}\end{array}$ & 32 & 34 & $\begin{array}{l}33 \% \\
780 \mathrm{~km}\end{array}$ \\
\hline Oct.-. & 32 & 37 & $34.5 \%$ & 29 & 34 & $\begin{array}{l}31.5 \% \\
1690 \mathrm{~km}\end{array}$ \\
\hline Nov.-. & 39 & 36 & $37.5 \%$ & 39 & 32 & $\begin{array}{l}35.5 \% \\
1910 \mathrm{~km}\end{array}$ \\
\hline Dec & 30 & 39 & $\begin{array}{l}34.5 \% \\
1850 \mathrm{~km}\end{array}$ & 34 & 36 & $\begin{array}{c}35 \% \\
1880 \mathrm{~km}\end{array}$ \\
\hline Mean & 36.5 & 39 & $2030 \mathrm{~km}$ & 34.5 & 40 & $1900 \mathrm{~km}$ \\
\hline
\end{tabular}

*Rough estimates - not included in the mean.

Table 1 shows that the distances between the first (in time) and second minima, and between the second and third minima are nearly the same both during sunrise and during sunset, but that there is a slight tendency for the distances to increase as the sunset or sunrise line approaches the region of the second and third minima. This tendency is possibly significant in the statistical sense. On the other hand, the apparent difference between the mean of the sunrise spacings and the mean of the sunset spacings is probably not statistically significant.

All the observations described above have been made by receiving at one point on each path. Some

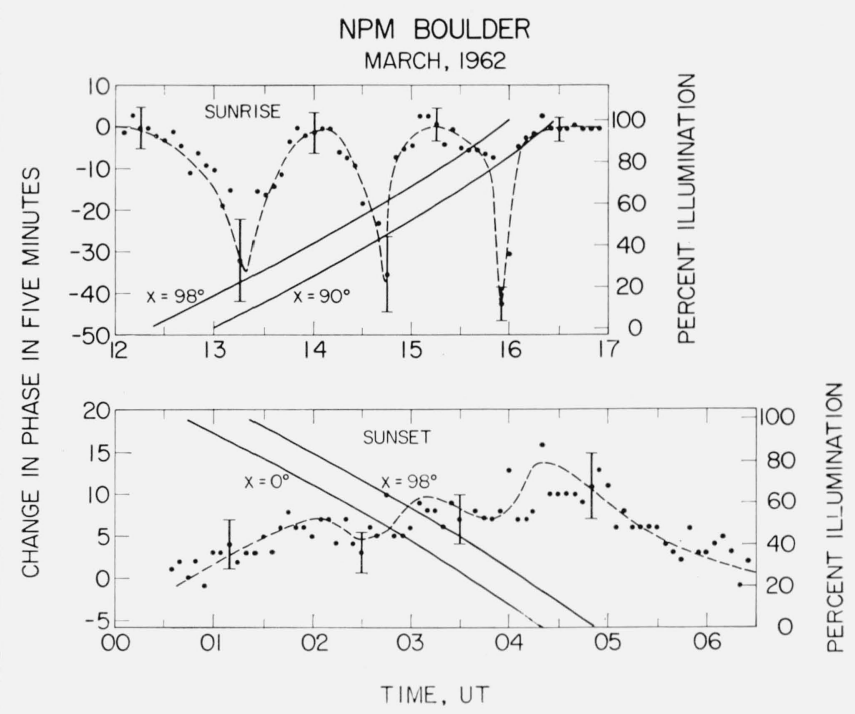

Figure 3. Mean rate of change of phase (degrees in $5 \mathrm{~min}$ ) during March 1962 for the NPM signal received at Boulder.

The solid lines show the percentage of the path illuminated by the sun. The heights of the vertical bars are twice the standard deviation of the means.

observations have been made in Washington, D.C., however, which to a very close approximation lies on the same great circle path from NPM, as Boulder, Colo. The few days' observations available, made during November 1962, show that the times of signal minima at sunrise are the same at both Boulder and Washington to within 5 min or less. This of course only applies after the sunrise line has passed Boulder.

Furthermore, the rate of change of phase and depth at fading are of comparable magnitude at the two points.

At sunset, on the other hand, it is not possible to detect fading on the records taken in Washington, although it is visible on the Boulder records. Limited data have also been obtained at Stanford University (by courtesy of $R$. A. Helliwell) near San Francisco with similar results at sunrise.

\section{Interpretation of the Observations}

As mentioned in the introduction it seems that an explanation based on the interference of two (or possibly more) waveguide modes is likely to be more satisfactory than one based on a single ray theory, particularly for paths as long as those being considered here. This approach is also suggested by the fact that the amplitude of the received signals reaches its minimum value at the same time as the rate of change of phase is maximum. Moreover, calculations by Wait [1962a] show that the second order mode may be excited with an amplitude comparable with that of the first order mode.

Let us consider the signal $E$ received by an antenna subject to the fields of two modes having guide wavelengths $\lambda_{1}$ and $\lambda_{2}$. The received signal $E$ at a distance 
$d$ from the source can be represented by

$$
E=E_{1} \cos \left(\omega t-\frac{2 \pi d}{\lambda_{1}}\right)+E_{2} \cos \left(\omega t-\frac{2 \pi d}{\lambda_{2}}+\varphi\right)
$$

where $E_{1}$ and $E_{2}$ are the amplitudes of the two modes and $\omega$ is the angular frequency of the wave. The phase angle $\varphi$ is included to take account of a possible difference in phase of the excitation factors of the two modes. If it is not zero, its effect is simply to uniformly translate along the path the positions of the minima and maxima of the standing wave pattern: thus it will be dropped in what follows.

If $E$ is written in the form

$$
E=E^{\prime} \cos \left(\omega t-\frac{2 \pi d}{\lambda_{1}}-\phi\right)
$$

then it is easily shown from (1) that

$$
\begin{aligned}
& E^{\prime}=\sqrt{E_{1}^{2}+2 E_{1} E_{2} \cos \theta+E_{2}^{2}} \\
& \phi=\tan ^{-1} \frac{E_{2} \sin \theta}{E_{1}+E_{2} \cos \theta}
\end{aligned}
$$

where

$$
\theta=2 \pi \lambda\left(\frac{1}{\lambda_{1}}-\frac{1}{\lambda_{2}}\right)
$$

The amplitude $E^{\prime}$ of the received signal is a minimum when $\theta=\pi, 3 \pi, 5 \pi$, etc., and $\frac{d \phi}{d \theta}$ is a maximum for the same values of $\theta$, in accordance with the observations. Thus as $d$ is varied points of minimum amplitude occur at distances which differ by $D$ where

$$
D=\frac{\lambda_{2} \lambda_{1}}{\lambda_{2}-\lambda_{1}} \simeq \frac{\lambda^{2}}{\lambda_{2}-\lambda_{1}}
$$

since the free space wavelength $\lambda \simeq \lambda_{2}$ or $\lambda_{1}$. Thus knowing $D$, it is possible to determine experimental values for $\lambda_{2}-\lambda_{1}$.

Expressions can be derived for $\lambda_{1}$ and $\lambda_{2}$, the guide wavelengths of the first and second order modes but the results are somewhat complicated when the spherical nature of the earth is included. Because of this, numerical values for $\lambda_{1}$ and $\lambda_{2}$ calculated by Wait [1962a] will be used. These calculations relate $\lambda, \lambda_{1}$ or $\lambda_{2}$ to the height of the ionosphere when the earth is spherical and perfectly conducting and the ionosphere is sharply bounded but of finite conductivity. It is possible to make allowance for the effect of the earth's magnetic field and for horizontal stratification of the ionosphere [Wait, 1962a] but these corrections are small and will be omitted in this discussion.

Using the values of $\lambda_{1}$ and $\lambda_{2}$ calculated by Wait [1962a] together with (2), the curves given in figure 4 for the frequencies of $18 \mathrm{kc} / \mathrm{s}(\mathrm{NBA})$ and 19.8 $\mathrm{kc} / \mathrm{s}$ (NPM) have been obtained. These show the relationship between the height of the ionosphere, $h$, under which the two modes are propagating and the distance, $D$, between the resulting interference minima for the two frequencies on the assumption that the interference pattern moves with the sunset line. Thus, the distances between minima given in table 1 for the NPM to Boulder path, and the corresponding distances for the NBA to Frankfurt path may be converted into equivalent ionosphere heights by plotting them on the appropriate curves which has been done in figure 4 .

The resulting heights from figure 4 appear to be reasonable and, although the scatter is somewhat large, it is to be noted that there is a considerable overlap of the heights deduced for the two paths which are being considered. It was noted earlier that the mean distances between minima at sunset and sunrise on the NPM-Boulder path were essentially equal. Thus it can be deduced that the mean heights of the ionosphere above the interference regions are also equal at sunrise and sunset.

It remains to establish whether the interference region is the nighttime or daylit portion of the path, or possibly both.

\section{Multimode Propagation Model}

The most direct and possibly the simplest approach to this question is to recognize that the two modes are excited by the transmitting antenna and propagate in both daytime and nighttime regions [Wait 1962a]. The modes travel with a phase velocity appropriate to the height of the earthionosphere waveguide in which they are propagating. When a sunrise or sunset line is encountered, the modes assume phase velocities determined by the new ionospheric height.

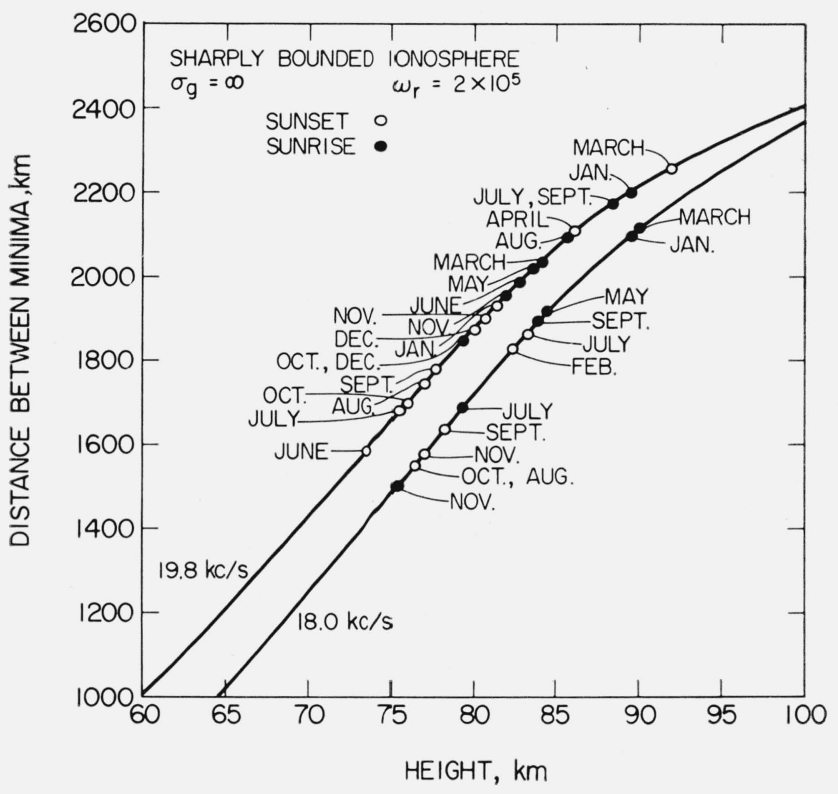

FiguRE 4. The relationship between the distance between minima, frequency and height of propagation. 
For example, consider the sunrise case illustrated in figure 5. At the receiver $R$ the phase delay $\theta_{1}$ of the first order mode is given by

$$
\theta_{1}=2 \pi \frac{d_{N}}{\lambda_{N 1}}+2 \pi \frac{d_{D}}{\lambda_{D 1}}
$$

while the phase delay $\theta_{2}$ of the second order mode is given by

$$
\theta_{2}=2 \pi \frac{d_{N}}{\lambda_{N 2}}+2 \pi \frac{d_{D}}{\lambda_{D 2}}
$$

Thus the signal $E$ observed at the receiver is

$$
\begin{aligned}
E & =E_{1} \cos \left(\omega t-\theta_{1}\right)+E_{2} \cos \left(\omega t-\theta_{2}\right) \\
& \equiv E^{\prime} \cos \left(\omega t-\theta_{1}-\phi\right)
\end{aligned}
$$

where

$$
E^{\prime}=\sqrt{E_{1}^{2}+2 E_{1} E_{2} \cos \left(\theta_{2}-\theta_{1}\right)+E_{2}^{2}}
$$

and

$$
\phi=\tan ^{-1} \frac{E_{2} \sin \left(\theta_{2}-\theta_{1}\right)}{E_{1}+E_{2} \cos \left(\theta_{2}-\theta_{1}\right)} .
$$

In these expressions, $E_{1}$ and $E_{2}$ are the amplitudes of the two modes at the receiver and are, in general, functions of distance. The possibility that the two modes are excited with differing phases is again neglected. Now

$$
\theta_{2}-\theta_{1}=2 \pi d_{N}\left(\frac{1}{\lambda_{N 2}}-\frac{1}{\lambda_{N 1}}\right)+2 \pi d_{D}\left(\frac{1}{\lambda_{D 2}}-\frac{1}{\lambda_{D 1}}\right)
$$

where $d$, the total path length, is equal to the sum of the lengths of the illuminated path $d_{D}$ and the dark path $d_{N}$.

$\theta_{2}-\theta_{1}=2 \pi\left\{d\left(\frac{1}{\lambda_{N 2}}-\frac{1}{\lambda_{N 1}}\right)\right.$

$$
\left.+d_{D}\left[\left(\frac{1}{\lambda_{D 2}}-\frac{1}{\lambda_{D 1}}\right)-\left(\frac{1}{\lambda_{N 2}}-\frac{1}{\lambda_{N 1}}\right)\right]\right\} \text {. }
$$

The first term is constant but the second term varies with the length of daylit path. In general $\left|\frac{1}{\lambda_{D 2}}-\frac{1}{\lambda_{D 1}}\right|>\left|\frac{1}{\lambda_{N 2}}-\frac{1}{\lambda_{N 1}}\right|$ Wait (1962a). Hence as the path changes from dark to light (increasing $d_{D}$ ) $\theta_{2}-\theta_{1}$ decreases in magnitude (i.e., increases negatively). From the equation for $\phi$ above:

$$
\frac{d \phi}{d\left(\theta_{2}-\theta_{1}\right)}=\frac{\frac{E_{2}}{E_{1}}\left[\frac{E_{2}}{E_{1}}+\cos \left(\theta_{2}-\theta_{1}\right)\right]}{1+2 \frac{E_{2}}{E_{1}} \cos \left(\theta_{2}-\theta_{1}\right)+\left(\frac{E_{2}}{E_{1}}\right)^{2}} .
$$

At the points of destructive interference cos $\left(\theta_{2}-\theta_{1}\right)=-1$. Thus

$$
\frac{d \phi}{d\left(\theta_{2}-\theta_{1}\right)}=\frac{-E_{2} / E_{1}}{1-E_{2} / E_{1}}
$$
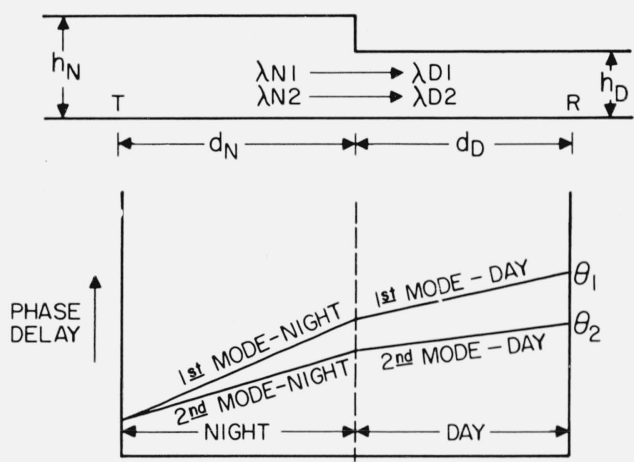

Figure 5. Multimode propagation in both the night and day region of the earth-ionosphere waveguide.

and if $E_{2} / E_{1}<1, \frac{d \phi}{d\left(\theta_{2}-\theta_{1}\right)}$ is negative.

Hence, as $d_{D}$ increases $\left(\theta_{2}-\theta_{1}\right)$ decreases and $\phi$ increases. Thus, during sunrise, $d_{D}$ increases and the phase delays at the points of signal minima increase. At sunset the same argument applies, $d_{D}$ decreases, $\theta_{2}-\theta_{1}$ increases and the phase delays decrease at the signal minima.

Both the above results are contrary to the experimental observations. Furthermore, it is easy to show with this model that at different points along the path the interference minima would occur at fixed distances behind the sunrise line. Hence the times of minima at sunrise would depend on the point at which observations were made, again in contradiction to the rather limited amount of available data. Because of this conflict between the observations, and the predictions of this simple model in which two modes are present at all times, the model seems quite inadequate.

An alternative model which appears to predict the observed results will now be discussed. It is assumed that, at sunrise, the two modes excited by the transmitter in the nighttime waveguide are converted at or near the sunrise boundary into two first order modes. These two first order modes are indistinguishable from each other and thus propagate as a single first order mode in the daytime waveguide. Some second order mode is bound to be excited in the daytime portion of the waveguide near the boundary, but because its attenuation rate is very high [Wait, 1962a] it can be ignored. At sunset, this first order mode is converted back into a first and a second order mode at the sunset boundary. Thus in the nighttime waveguide two modes are present whereas only one is present during the day. The situation is illustrated diagrammatically in figure 6 .

In the sunrise case the two modes $E_{1}$ and $E_{2}$ are propagating in the dark portion of the earthionosphere waveguide. At the sunrise line they can 


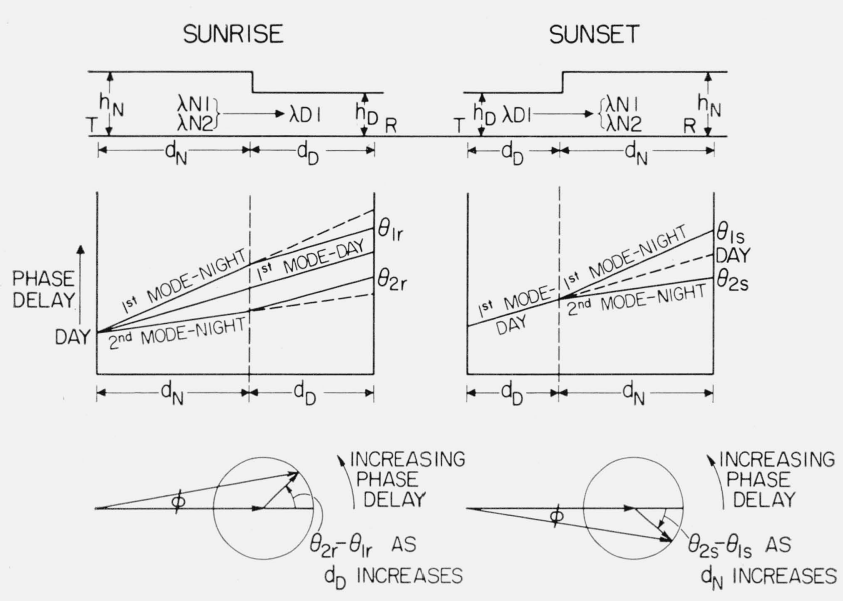

FIGURE 6. The assumed model which appears to explain the observations.

Note that multimode propagation only occurs in the nighttime region of the waveguide.

be expressed in the form

$$
\begin{aligned}
& E_{1}=E \cos \left(\omega t-2 \pi \frac{d_{N}}{\lambda_{N 1}}\right) \\
& E_{2}=E \cos \left(\omega t-2 \pi \frac{d_{N}}{\lambda_{N 2}}\right) .
\end{aligned}
$$

After passing the sunrise line, $E_{1}$ is essentially unchanged and produces a signal $E_{R 1}$ at the receiver, whose guide wavelength is $\lambda_{D 1}$, where

$E_{R 1}=a E \cos \left(\omega t-2 \pi \frac{d_{N}}{\lambda_{N 1}}-2 \pi \frac{d_{D}}{\lambda_{D 1}}\right)=a E \cos \left(\omega t-\theta_{R 1}\right)$

and $a$ is a constant which includes the effects of the change in height of the ionosphere on amplitude. The second order mode $E_{2}$ is however converted into a first order mode, which, to the east of the shadow line also has a guide wavelength $\lambda_{D 1}$. This converted mode produces a signal at the receiver given by

$E_{R 2}=b E \cos \left(\omega t-2 \pi \frac{d_{N}}{\lambda_{N 2}}-2 \pi \frac{d_{D}}{\lambda_{D 1}}\right)=b E \cos \left(\omega t-\theta_{R 2}\right)$.

Here $b$ is a conversion factor.

In general, both $E_{R 1}$ and $E_{R 2}$ will depend on distance, but this is not of real importance here and has been tacitly ignored.

The total field $E_{R}^{\prime}$ at the receiver is $E_{R 1}+E_{R 2}$ which, as before, can be written in the form

$$
E_{R}=E_{R} \cos \left(\omega t-\theta_{R 1}-\phi_{R}\right)
$$

where

$$
\begin{gathered}
E_{R}=E \sqrt{a^{2}+2 a b \cos \theta_{R}+b^{2}} \\
\phi_{R}=\tan ^{-1} \frac{b \sin \theta_{R}}{a+b \cos \theta_{R}}
\end{gathered}
$$

and

$$
\begin{aligned}
\theta_{R} & =\theta_{R 2}-\theta_{R 1}=2 \pi d_{N}\left(1 / \lambda_{N 2}-1 / \lambda_{N 1}\right) \\
& =2 \pi d_{D}\left(1 / \lambda_{N 1}-1 / \lambda_{N 2}\right)-2 \pi d\left(1 / \lambda_{N 1}-1 / \lambda_{N 2}\right) .
\end{aligned}
$$

Now $\lambda_{N 2}>\lambda_{N 1}$ and as daylight advances over the path, $d_{D}$ and $\theta_{R}$ increase (i.e., become more positive). Thus, as before, at the signal minima $\cos \theta_{R}=-1$ and $\phi_{R}$ decreases. This result can be seen from the phasor diagram shown in figure 6 . The amplitude at the receiver $E_{R}$ is given by (3) and thus depends on $\theta_{R}$. Equation (4) shows that the amplitude depends only on $d_{N}$, the dark portion of the path, and not on the total length of the path. Thus it would be expected that the signal minima would occur simultaneously to the east of the sunrise line (in the case of west-to-east transmission).

Thus it can be seen that for the sunrise case, this model explains both the direction of the observed rapid phase change during the signal minima, and the observed apparent simultaneity of fading to the east of the sunrise line.

The assumed situation at sunset for west-to-east propagation is also shown in figure 6 . In the daytime portion of the waveguide, a single first order mode is propagating. At the sunset line this is converted into a new first order mode $E_{S 1}$ together with a second order mode $E_{S 2}$ : these two modes then interfere as they travel along the nighttime waveguide.

The signal at the receiver due to the first order mode can be written as

$$
E_{S 1}=f E \cos \left(\omega t-\frac{2 \pi d_{D}}{\lambda_{D 1}}-\frac{2 \pi d_{N}}{\lambda_{N 1}}\right)=f E \cos \left(\omega t-\theta_{S 1}\right)
$$

while that due to the second order mode is

$$
E_{S 2}=g E \cos \left(\omega t-\frac{2 \pi d_{D}}{\lambda_{D 1}}-\frac{2 \pi d_{N}}{\lambda_{N 2}}\right)=g E \cos \left(\omega t-\theta_{S 2}\right)
$$

where $f$ and $g$ are conversion coefficients and $d_{D}, d_{N}$ are the lengths of path which are daylit and dark respectively.

Once more the combined signal can be written as

$$
E_{S}^{\prime}=E_{S} \cos \left(\omega t-\theta_{S 1}-\phi_{S}\right)
$$

where

$$
\begin{gathered}
E_{S}=E \sqrt{f^{2}+2 f g \cos \theta_{S}+g^{2}} \\
\phi_{S}=\tan ^{-1} \frac{g \sin \theta_{S}}{f+g \cos \theta_{S}}
\end{gathered}
$$

and

$$
\theta_{S}=\theta_{S 2}-\theta_{S 1}=2 \pi d_{N}\left(1 / \lambda_{N 2}-1 / \lambda_{N 1}\right) .
$$

Again $\lambda_{N 2}>\lambda_{N 1}$ and as $d_{N}$ increases $\theta_{S}$ becomes more negative. Thus, using the earlier arguments, $\phi_{S}$ increases at the time of signal minima. This again is in accordance with the observed behavior at sunset on west-to-east path. 
In this particular case, the interference pattern is "attached" to the dark side of the sunset line and moves westward with it. This suggests that signal minima at different points along a great circle path will occur at different times, in contrast to the sunrise case. It has not been possible to confirm this, because of the lack of adequate data.

\section{Discussion}

In the above explanation the effect of path attenuation has been ignored. It is to be expected, in daytime at least, that the attenuation rate of the second order mode will be much greater than that for the first [Wait 1962a]. Thus the depth of fading at sunrise should increase as the shadow line approaches the transmitter, in the two cases considered in this paper. This is in agreement with the experimental observations.

At sunset, by the same argument, the fading should be deepest when the shadow line is closest to the receiver. The observations do not confirm this deduction, particularly for the NBA-Frankfurt path. This is possibly because of changes in the angle between the path direction and the sunset line, or because mode conversion does not take place entirely at the sunset line. These points require further investigation. Nevertheless, the Washington, D.C., observations on NPM show that the sunset depth of fading is less than at Boulder at the same time, a result to be expected if the interfering modes occur along the nighttime portion of the path and are attenuated.

Estimates of the ionospheric heights of the region in which multimode propagation occurs have been given in figure 4 . It was earlier mentioned that there is no significant difference between the heights determined from the sunrise and sunset variations. This is in accordance with the explanation developed above, which shows that the heights should be the same, and are the heights of the nighttime ionosphere. It must be remembered however, that what is actually determined is the difference in guide wavelengths of the two modes. These are then used to deduce the effective height of the ionosphere, using mode theory. In this paper, the effect of stratification of the ionosphere and effects due to the magnetic field have not been considered. If these are included, deduced heights of the ionosphere under nighttime conditions could be slightly different from those shown in figure 4.

The deductions in this paper are based on observations made on two long west-to-east paths. However, some data obtained in Hawaii on the signals from NBA have also been examined, but not in detail. This path is essentially east to west; thus at sunrise, propagation is from daylight into darkness, while at sunset the reverse holds. If this is kept in mind, the results of the preliminary examination of the data do not conflict with the deductions made in this paper.

A major question is the mechanism which converts the two modes excited by the transmitter in the nighttime waveguide into a single mode in the day- time portion of the waveguide and subsequently converts this single mode back into two modes at or near the sunset line. Wait [1962b], has made a preliminary analysis of this problem and finds that mode conversion at a gently tapered transition is very small. Later work [Wait 1963b] however, indicates that conversion may be significant for more rapid transitions. It is interesting to note that the depth of fading at sunrise is much greater than at sunset. This is presumably because the interfering second order mode is directly excited by the transmitter, when it is in the nighttime portion of the path. However, since the second order mode is converted into one of first order over a very short distance and with considerable efficiency, one might expect the converse to be true at sunset. Because of the smaller depth of fading at sunset, this is apparently not the case. Thus, there is a difference between the mode conversion efficiencies at sunrise and sunset which is possibly related to the fact that the height transition is sharper at sunrise (because of photodetachment) than at sunset. It is clear that further work needs to be done on the whole problem of mode conversion.

\section{Conclusions}

One year's observations of the periodic variations of the phase and amplitude which occur on the VLF signals from NBA and NPM observed at Frankfurt and Boulder have been examined. The major features of the fading are

1. The fading is much more pronounced at sunrise than at sunset.

2. The sunrise observations show five amplitude minima on the NBA-Frankfurt path and three on the NPM-Boulder path.

3. The rate of change of phase is a maximum at the times when the signal is a minimum, very approximately.

4. The direction of the rapid phase changes at the signal minima is in the same direction as the prevailing phase change; i.e., a decrease in phase delay at sunrise and an increase at sunset.

5. At sunrise the depth of fading increases as the sunrise line approaches the transmitter.

6. Limited observations at two points along the same great circle path from the transmitter indicate that fading occurs simultaneously and is of constant depth on the daylight side of the sunrise line.

7. At sunset, the depth of fading is smaller at the eastern end of the path.

8. Interpretation of the fading as modal interference leads to the conclusion that the ionospheric heights where the interference occurs are the same at sunrise and at sunset. It is later found that these heights are nighttime heights.

9. There is some evidence which suggests that the depth of fading is dependent on the angle between the great circle path and the sunset (or sunrise line).

The first eight of these experimental results can be explained if it is recognized that at sunrise the transmitter (which is in darkness) excites a first and a 
second order mode. Because of the difference in the phase velocity of these modes, an interference pattern is produced. At the sunrise line, it is suggested that the two modes are converted into one of first order whose amplitude is proportional to the sum of the two modes on the dark side of the sunrise line. At sunset the first order mode in the daytime portion of the earth-ionosphere waveguide is assumed to be converted into a first and a second order mode which propagate in the nighttime portion of the guide, and again produce an interference pattern. As the sunrise or sunset line moves along the path, the interference patterns cause the periodic amplitude and phase variation which are observed experimentally.

The main problem with this explanation is in the details of the mode conversion at the shadow line. Although recent work by Wait [1963b] suggests that higher order modes will be produced at a discontinuity in the height of the earth-ionosphere waveguide, further work is required to account for the observed magnitude of the mode conversion factors.

The writer expresses his appreciation to Anabeth Murphy for completing the onerous task of scaling the experimental records, and to Allen H. Brady for devising computer methods of reducing the scaled data. The Frankfurt observations were made by the Battelle Institute. This work was supported in part by the Advanced Research Projects Agency.

\section{References}

Budden, K. G. (1961), The waveguide mode theory of wave propagation (Prentice-Hall, Inc., New York, N.Y.).

Pierce, J. A. (1957), Intercontinental frequency comparisons by VLF radio transmission, Proc. IRE 45, No. 6, 794.

Rieker, J. (Mar-Apr. 1963), Sunset and sunrise in the ionosphere: effects on the propagation of longwaves, J. Res. NBS 67D (Radio Prop.), No. 2, 119-138.

Wait, נ. R., and L. B. Perry (1957), Calculations of ionospheric reflection coefficients at very low frequencies, J. Geophys Res. 62, No. 1, 43.

Wait, J. R. (1962a), Electromagnetic waves in stratified media (Pergamon Press, Itd., Oxford).

Wait, J. R. (July-Aug. 1962b), An analysis of VLF mode propagation for a variable ionosphere height, J. Res. NBS 66D (Radio Prop.), No. 4, 453-461.

Wait, J. R. (Jan. 1, 1963a), A note on diurnal phase changes of VLF waves for long paths, J. Geophys Res. 68, 338-340.

Wait, J. R. (1963b), VLF propagation in the earth-ionosphere waveguide of nonuniform width, The Ionosphere (Institute of Physics and the Physical Society, London), p. 446.

Yokoyama, E., and I. Tanimura (1933), Some long distance transmission phenomena of low frequency waves, Proc. IRE 21, No. 2, 263.

(Paper 68D1-313) 